\title{
THE EFFECT OF ELASTIC AND INELASTIC SCATTERING ON ELECTRONIC TRANSPORT IN OPEN SYSTEMS
}

\author{
KARol KUlinOWSKI $^{a}$, MACIEJ WOŁOSZYN $^{a}$, MARTA RADECKA $^{b}$, BARTŁOMIEJ J. SPISAK ${ }^{a, *}$
}

\author{
${ }^{a}$ Faculty of Physics and Applied Computer Science \\ AGH University of Science and Technology, al. Mickiewicza 30, 30-059 Cracow, Poland \\ e-mail: bj.s@agh.edu.pl \\ ${ }^{b}$ Faculty of Materials Science and Ceramics \\ AGH University of Science and Technology, al. Mickiewicza 30, 30-059 Cracow, Poland
}

\begin{abstract}
The purpose of this study is to apply the distribution function formalism to the problem of electronic transport in open systems, and to numerically solve the kinetic equation with a dissipation term. This term is modeled within the relaxation time approximation and contains two parts, corresponding to elastic or inelastic processes. The collision operator is approximated as a sum of the semi-classical energy dissipation term and the momentum relaxation term, which randomizes the momentum but does not change the energy. As a result, the distribution of charge carriers changes due to the dissipation processes, which has a profound impact on the electronic transport through the simulated region discussed in terms of the current-voltage characteristics and their modification caused by the scattering. Measurements of the current-voltage characteristics for titanium dioxide thin layers are also presented, and compared with the results of numerical calculations.
\end{abstract}

Keywords: kinetic equation, relaxation time approximation, scattering processes.

\section{Introduction}

Transport processes pose a wide variety of problems which are interesting for fundamental research in different branches of physics and engineering. Particularly, a lot of attention is devoted to electronic transport in solid state systems because they are used as elements of common electronic devices that are subject to progressive miniaturization. Simultaneously, more and more functionality of these devices is expected. These needs stimulate the search for new materials, as well as the development of theoretical and computational techniques or simulations for exploring transport phenomena of particular relevance in these systems.

A full theoretical description of electronic transport in the systems considered should be based on the methods of non-equilibrium statistical mechanics, but such description is an extremely complicated problem (Fujita, 1966; Danielewicz, 1984; Rammer, 2007; Schieve and Horwitz, 2009). Therefore, some physical assumptions

\footnotetext{
*Corresponding author
}

and approximations are usually applied to simplify this issue (Di Ventra, 2008; Ferry et al., 2009). One of the most common assumptions is to treat the studied system as an active element of the device connected to large electrodes, which play the role of reservoirs of charge and heat. Each of these electrodes is characterized by its own equilibrium distribution function in the Fermi-Dirac form that is specified through the temperature and the chemical potential. For a fixed temperature, the difference between the chemical potentials associated with reservoirs is proportional to the bias voltage which produces the electric field across the studied system and the current of electrons flowing through the system is generated. The real structure of the system can be defected or can contain dopants; moreover, a collective thermal vibration of ions generates phonons which interact with the conduction electrons.

All these factors have a significant impact on the electronic transport, and separation of contributions from the different scattering mechanisms is the first step to understand the transport properties of the system. In many 
interesting cases, it is difficult to find some analytical solution for the transport problem considered in solid state nanostructures. Hence, great effort is focused on numerical solutions. For example, considerable attention has been devoted to improving the numerical methods of solving the classical as well as the quantum kinetic equation. Efficient numerical schemes based on non-uniform meshes are developed for deterministic discretization method (Kim, 2007; Costolanski and Kelley, 2010; Kim and Kim, 2015; Schulz and Mahmood, 2016) or techniques based on the fast Fourier transform via the split-operator method. (Gómez et al., 2014; Spisak et al., 2015; Cabrera et al., 2015; Thomann and Borz, 2017). Besides, stochastic methods based on different variants of the Monte Carlo techniques are available (Jacoboni et al., 2001; Nedjalkov et al., 2004; Querlioz and Dollfus, 2010; Muscato and Wagner, 2016). On the other hand, numerical solutions for the Boltzmann equation are well established and documented (Nedjalkov and Vitanov, 1989; Nedjalkov et al., 2013; Hong et al., 2011; Hong and Jang, 2018; Jacoboni et al., 1988; Chatterjee et al., 2014; Sellier et al., 2014; Sellier and Dimov, 2014; Ben Abdallah et al., 1996).

The aim of this paper is to study the influence of scattering processes of carriers in a highly defected semiconductor device on its transport characteristics. In such systems, the momentum of the carriers is randomized due to scattering processes and their energy can be changed as a result of interaction with phonons. For this reason we solve the kinetic equation with a dissipation term modeled with the relaxation time approximation. Within this approximation we take into account that the dissipation term consists of two parts. One describes the momentum relaxation due to elastic scattering, and the other describes the momentum and energy relaxation due to inelastic scattering processes.

The paper is organized as follows. In Section 2 we present the derivation of the kinetic equation for the distribution function, starting from the modified form of the von Neumann equation for the density operator. The intermediate step for deriving the kinematic equation is based on the Weyl transform and the Wigner function. Therefore, we briefly present selected properties of the Wigner function, which is usually interpreted as the quasi-distribution function. It is closely related to the classical distribution function through the averaging procedure, which destroys the quantum interference. Further, we discuss different approximations which are usually made for simplification of full quantum theory of electronic transport. Section 3 contains a model of the semiconductor defected system and the discussion of the numerical method that is used to solve the presented problem. In turn, in Section 4 we present the results of numerical calculations and measurements which support our theoretical analysis of transport properties of the model system. The measurements of transport characteristics of films made of titanium dioxide, i.e., the current-voltage characteristics, are performed at room temperature. In addition, a discussion of the obtained theoretical as well as experimental results is included here. This report is concluded in Section 5, where we summarize the presented results.

\section{Theory}

It is widely recognized that the starting point for quantum theory of electronic transport in solid-state systems is the von Neumann equation (e.g., Kohn and Luttinger, 1957; Luttinger and Kohn, 1958),

$$
i \hbar \frac{\mathrm{d} \hat{\rho}(t)}{\mathrm{d} t}=\left[\hat{H}_{0}, \hat{\rho}(t)\right]
$$

where $\hat{H}_{0}$ is the one-particle Hamiltonian of the unperturbed system in the form

$$
\hat{H}_{0}=\hat{p}^{2} / 2 m+U(\hat{x}) ;
$$

here $\hat{p}$ denotes the momentum operator and $U(\hat{x})$ is the potential energy operator of carriers with the effective mass $m$. In turn, $\hat{\rho}(t)$ is the one-particle density operator defined by the formula (Ter Haar, 1961)

$$
\hat{\rho}(t)=\sum_{n} p_{n}\left|\phi_{n}(t)\right\rangle\left\langle\phi_{n}(t)\right|
$$

where $p_{n}$ corresponds the probability of finding the system in the pure state $\left|\phi_{n}(t)\right\rangle$. The density operator can be characterized by its own matrix elements which form the density matrix. For example, the density operator in the position representation is

$$
\rho\left(\xi, \xi^{\prime}\right)=\left\langle\xi|\hat{\rho}(t)| \xi^{\prime}\right\rangle .
$$

The diagonal elements of the density matrix represent the electron's density whereas its off-diagonal elements are responsible for the phase correlations of electrons. Let us note that the application of the von Neumann equation to the description of the conduction electrons dynamics in the systems considered is equivalent to the assumption that the electronic transport is coherent, i.e., quantum interference becomes an important ingredient of the dynamical process description. On the other hand, this coherent dynamics of conduction electrons are often perturbed by different kinds of uncontrollable interactions which destroy the phase coherence of the carriers. One of the examples of this situation in real systems is the interaction of the carriers gas with the phonon gas. In such a case, one can observe the transfer of energy or momentum between these subsystems. This transfer is expressed in terms of an inelastic or elastic scattering ratio, depending on the responsible physical mechanism. 
In fact, this is a statistical process which introduces some kind of irreversibility to the system, resulting in dissipation. These considerations lead to the conclusion that Eqn. (1) should contain an additional term which introduces dissipation. On this basis, the von Neumann equation is transformed to the form

$$
i \hbar \frac{\mathrm{d} \hat{\rho}(t)}{\mathrm{d} t}=\left[\hat{H}_{0}, \hat{\rho}(t)\right]+\hat{D}[\hat{\rho}(t)]
$$

where the operator $\hat{D}[\hat{\rho}(t)]$ represents the dissipative term. The form of this term is a matter of wide dispute, and several discussions have been reported for this approach (e.g., Chruściński and Pascazio, 2017).

The phase space representation of Eqn. (5) can be derived in two steps. First of all, we write Eqn. (5) in the position representation $\left[\xi-\xi^{\prime}\right]$, namely,

$$
\begin{aligned}
& \frac{\partial}{\partial t} \rho\left(\xi^{\prime}, \xi, t\right) \\
& =-\frac{\hbar}{2 m i}\left(\frac{\partial^{2}}{\partial \xi^{\prime 2}}-\frac{\partial^{2}}{\partial \xi^{2}}\right) \rho\left(\xi^{\prime}, \xi, t\right) \\
& \quad+\frac{1}{i \hbar}\left[U\left(\xi^{\prime}\right)-U(\xi)\right] \rho\left(\xi^{\prime}, \xi, t\right)+\left\langle\xi^{\prime}|\hat{D}[\hat{\rho}(t)]| \xi\right\rangle .
\end{aligned}
$$

Then we introduce an auxiliary position representation $[x-X]$ using the canonical variables transformation in the form

$$
\left\{\begin{array}{l}
x=\frac{1}{2}\left(\xi^{\prime}+\xi\right), \\
X=\xi^{\prime}-\xi
\end{array}\right.
$$

New variables $x$ and $X$ are introduced as the center-of-mass and relative position coordinates, respectively. Straightforward application of the coordinate transformation given by Eqn. (7) to Eqn. (6) leads to the following form of the equation of motion for the density matrix:

$$
\begin{aligned}
& \frac{\partial}{\partial t} \rho\left(x+\frac{1}{2} X, x-\frac{1}{2} X, t\right) \\
& =-\frac{\hbar}{m i} \frac{\partial^{2}}{\partial x \partial X} \rho\left(x+\frac{1}{2} X, x-\frac{1}{2} X, t\right) \\
& \quad+\frac{1}{i \hbar}\left[U\left(x+\frac{1}{2} X\right)-U\left(x-\frac{1}{2} X\right)\right] \\
& \quad \times \rho\left(x+\frac{1}{2} X, x-\frac{1}{2} X, t\right) \\
& \quad+\left\langle x+\frac{1}{2} X|\hat{D}[\hat{\rho}(t)]| x-\frac{1}{2} X\right\rangle .
\end{aligned}
$$

The mixed position-momentum representation $[x-p]$ is obtained by the application of the Fourier transform

$$
\begin{aligned}
g(X) & =\frac{1}{2 \pi \hbar} \int \mathrm{d} p g(p) \exp \left[\frac{i}{\hbar} p X\right], \\
g(p) & =\int \mathrm{d} X g(X) \exp \left[-\frac{i}{\hbar} p X\right]
\end{aligned}
$$

to Eqn. (8), and the definition of the Wigner function (Wigner, 1932; Tatarskiı̆, 1983; Lee, 1995; Schleich, 2001),

$$
\begin{aligned}
& \varrho(x, p, t) \\
& =\frac{1}{2 \pi \hbar} \int \mathrm{d} X \rho\left(x+\frac{X}{2}, x-\frac{X}{2}, t\right) \\
& \quad \times \exp \left[-\frac{i p X}{\hbar}\right] .
\end{aligned}
$$

This is a special form of the inverse Weyl transform (Leaf, 1968). The Wigner function plays a similar role as the distribution function in the classical statistical mechanics, in the sense that the WDF can be used to calculate the expectation value of any dynamical variable as follows:

$$
\langle A(t)\rangle=\int \mathrm{d} x \mathrm{~d} p A_{W}(p, x) \varrho(x, p ; t)
$$

where $A_{W}(p, x)$ is the Weyl symbol of the quantum-mechanical operator of a dynamical variable $\hat{A}$ in the position representation,

$$
\begin{aligned}
A_{W}(p, x)= & \int \mathrm{d} X\left\langle x+\frac{X}{2}|\hat{A}(\hat{p}, \hat{x})| x-\frac{X}{2}\right\rangle \\
& \times \exp \left[-\frac{i p X}{\hbar}\right] .
\end{aligned}
$$

Furthermore, the Wigner function is a real and normalized function,

$$
\int \mathrm{d} x \varrho(x, p ; t)=1,
$$

and its marginals

$$
n(x, t)=\int \mathrm{d} p \varrho(x, p ; t)
$$

and

$$
\eta(p, t)=2 \pi \hbar \int \mathrm{d} x \varrho(x, p ; t)
$$

are interpreted as position and momentum distributions, respectively. The Wigner function cannot take on arbitrarily large values, i.e., there exists an upper bound for this function in the form

$$
|\varrho(x, p ; t)|=\frac{1}{\pi \hbar} .
$$

There is another interesting property of the Wigner function, namely, it can take on negative values in some region of the phase space. This property makes it impossible to interpret the Wigner function as a classical probability distribution. From the physical standpoint this is evident because the position and momentum cannot have simultaneously defined values, due to the uncertainty principle. Nevertheless, the negative values of the Wigner 
function are a hallmark of non-classical properties of the state which is represented by the function. The classical limit of the Wigner function should be consistent with the classical distribution function $f(x, p, t)$. This expectation can be realized owing to the averaging procedure of the Wigner function over the phase-space area $\Delta x \Delta p$ larger than the elementary quantum cell $2 \pi \hbar$. This guarantees that the quantum interference effects which are coded in the negative part of the Wigner function vanish and the corresponding function is always non-negative in the phase space. In this sense the expression

$$
\begin{aligned}
& \int_{\Delta x \Delta p} \mathrm{~d} x \mathrm{~d} p \varrho(x, p, t) \\
= & \int_{\Delta x \Delta p} \mathrm{~d} x \mathrm{~d} p f(x, p, t)+O\left[\left(\frac{2 \pi \hbar}{\Delta x \Delta p}\right)^{2}\right]
\end{aligned}
$$

illustrates the fact that the Wigner function in the classical limit $\Delta x \Delta p \gg 2 \pi \hbar$ can be regarded as the classical distribution function.

Returning to the kinetic equation, we can express the equation of motion corresponding to Eqn. (8) in the mixed representation as follows:

$$
\begin{aligned}
& \frac{\partial \varrho(x, p, t)}{\partial t}+\frac{p}{m} \frac{\partial \varrho(x, p, t)}{\partial x} \\
& =\frac{1}{\hbar^{2}} \int \mathrm{d} p^{\prime} W\left(x, p-p^{\prime}\right) \varrho\left(x, p^{\prime}, t\right) \\
& \quad+D_{W}[\varrho(x, p, t)],
\end{aligned}
$$

where $D_{W}[\varrho(x, p, t)]$ is the Weyl symbol of the dissipation term and the integral kernel $W\left(x, p-p^{\prime}\right)$ is the so-called Wigner potential. Its explicit form for a given potential energy $U(x)$ is given by the formula

$$
\begin{aligned}
& W\left(x, p-p^{\prime}\right) \\
& =\frac{1}{2 \pi i} \int \mathrm{d} X\left[U\left(x+\frac{X}{2}\right)-U\left(x-\frac{X}{2}\right)\right] \\
& \quad \times \exp \left[-\frac{i\left(p-p^{\prime}\right) X}{\hbar}\right] .
\end{aligned}
$$

The Wigner potential is a real-valued function, anti-symmetric with respect to the momentum. We note that the potential at the point $x$ is defined by the Fourier transform of the central difference of the potential energy around $x$. In fact, this means that the Wigner potential represents the non-local potential which incorporates the quantum-mechanical effects such as quantum interference into the dynamics of the Wigner function, making it negative in some regions of the phase space. Assuming that the potential energy is a slowly varying function in the real space, we can develop the potential energy $U(x \pm X / 2)$ into the power series around $x$ up to the linear order,

$$
U\left(x \pm \frac{1}{2} X\right) \approx U(x) \pm \frac{1}{2} \frac{\mathrm{d}}{\mathrm{d} x} U(x) X
$$

which is equivalent to the gradient approximation (Mahan, 2000; Di Ventra, 2008). This kind of approximation is consistent with the general request that the kinetic equation for the classical distribution function should take into account only the slow component of the spatial variations due to the perturbation while its fast component should be eliminated by an adequate procedure (Mahan, 2000). Using this result for the energy potential difference appearing in Eqn. (19), we obtain

$$
U\left(x+\frac{1}{2} X\right)-U\left(x-\frac{1}{2} X\right) \approx \frac{\mathrm{d}}{\mathrm{d} x} U(x) X .
$$

Hence, the Wigner potential takes on the form

$$
W\left(x, p-p^{\prime}\right)=\frac{1}{2 \pi i} \int \mathrm{d} X \frac{\mathrm{d}}{\mathrm{d} x} U(x) X e^{-i / \hbar\left(p-p^{\prime}\right) X} .
$$

One might notice that

$$
\frac{1}{i} X e^{-i / \hbar\left(p-p^{\prime}\right) X}=\hbar \frac{\partial}{\partial p}\left[e^{-i / \hbar\left(p-p^{\prime}\right) X}\right],
$$

and after some manipulations the Wigner potential can be written in the form

$$
W\left(x, p-p^{\prime}\right)=\hbar \frac{\mathrm{d}}{\mathrm{d} x} U(x) \frac{\partial}{\partial p}\left[\delta\left(p-p^{\prime}\right)\right] .
$$

This form of the non-local Wigner potential is then substituted into the first term on the right-hand side of Eqn. (18), i.e., into the so-called drift-term, which in the classical limit can be identified as a classical force. As a result, we obtain

$$
\begin{aligned}
& \frac{1}{\hbar^{2}} \int \mathrm{d} p^{\prime} \varrho\left(x, p^{\prime}, t\right) W\left(x, p-p^{\prime}\right) \\
& \approx \frac{1}{\hbar^{2}} \int \mathrm{d} p^{\prime} \varrho\left(x, p^{\prime}, t\right) \hbar^{2} \frac{\mathrm{d}}{\mathrm{d} x} U(x) \frac{\partial}{\partial p}\left[\delta\left(p-p^{\prime}\right)\right] \\
& =\frac{\mathrm{d}}{\mathrm{d} x} U(x) \frac{\partial}{\partial p}\left[\int \mathrm{d} p^{\prime} \varrho\left(x, p^{\prime}, t\right) \delta\left(p-p^{\prime}\right)\right] \\
& =\frac{\mathrm{d}}{\mathrm{d} x} U(x) \frac{\partial}{\partial p} \varrho(x, p, t) .
\end{aligned}
$$

The resulting formula has exactly the same form as the driving term in the Liouville equation, i.e., the factor

$$
-\frac{\mathrm{d}}{\mathrm{d} x} U(x)=F(x)
$$

can be interpreted as a force in classical mechanics. 


\section{Model and the method of calculation}

We consider a model of thin film which is connected to the source and drain electrodes as shown in Fig. 1 We assume that the simulation domain of the nanosystem, which includes the whole region of the thin film between the electrodes, is structurally defected, so that it can be described as a disordered system within the Gaussian model (Lifshits et al., 1987). This means that the average disorder potential is equal to zero, and the second cumulant of the potential is a function of the relative position of $x$ and $x^{\prime}$, i.e.,

$$
\langle U(x)\rangle=0 \quad \text { and } \quad\left\langle U(x) U\left(x^{\prime}\right)\right\rangle=K\left(x-x^{\prime}\right),
$$

where the symbol $\langle\cdot\rangle$ stands for the space average, and $K\left(x-x^{\prime}\right)$ is the potential correlator which corresponds to a macroscopically homogeneous and isotropic system.

As stems from Eqn. (21), the central difference of the energy potentials is proportional to the gradient. Using this gradient approximation, we can write the drift term in the classical limit as follows:

$\frac{1}{\hbar^{2}} \int \mathrm{d} p^{\prime} W\left(x, p-p^{\prime}\right) \varrho\left(x, p^{\prime}, t\right) \approx \frac{\mathrm{d} U(x)}{\mathrm{d} x} \frac{\partial}{\partial p} f(x, p, t)$.

Therefore, the classical limit of the Wigner equation (18) takes on the Boltzmann-like form, namely,

$$
\begin{aligned}
& \frac{\partial f(x, p, t)}{\partial t}+\frac{p}{m} \frac{\partial f(x, p, t)}{\partial x} \\
& -\frac{\partial U(x)}{\partial x} \frac{\partial f(x, p, t)}{\partial p}=D_{W}[f(x, p, t)] .
\end{aligned}
$$

The Weyl symbol of the dissipation term, $D_{W}[f(x, p, t)]$, is still a difficult issue, because its explicit form is unknown, although some results for the Caldeira-Leggett model (Caldeira and Leggett, 1981) were already discussed (Zurek, 2003).

In this report, we consider the stationary solution $f(x, p)$ of Eqn. 29) in the limit of a slowly-varying potential. For this purpose we apply the simplest model for the dissipation term which is known as the relaxation time approximation (Di Ventra, 2008; Ferry et al., 2009). In accordance with the work of Jonasson and Knezevic (2015), we choose this term to be approximately equal,

$$
\begin{aligned}
D_{W}[f(x, p)] \approx & -\gamma_{R}\left[f(x, p)-f^{e q}(p)\right] \\
& -\gamma_{M}[f(x, p)-f(x,-p)],
\end{aligned}
$$

where $\gamma_{R}$ and $\gamma_{M}$ are the strengths of inelastic and elastic scattering, respectively, and $f^{e q}(p)$ is the equilibrium distribution function.

The first term on the right-hand side of Eqn. (30) describes relaxation processes in which the momentum as well as energy are changed during the scattering. In

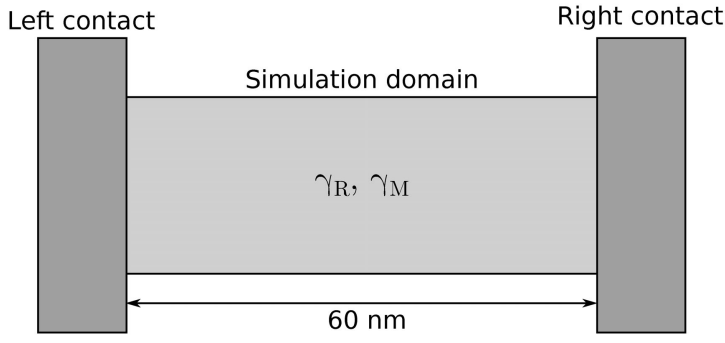

Fig. 1. Scheme of the thin film of length $L=60 \mathrm{~nm}$ (simulation domain) which is characterized by the rate of inelastic and elastic scattering processes, $\gamma_{R}$ and $\gamma_{M}$, respectively, and connected to the source (left) and drain (right) electrodes.

turn, the second term describes situations in which only the carrier's momentum is changed, whereas the energy is conserved. Taking into account all the above assumptions, we can finally write the kinetic equation in the following form:

$$
\begin{aligned}
\frac{p}{m} \frac{\partial f(x, p)}{\partial x}= & -\gamma_{R}\left[f(x, p)-f^{e q}(p)\right] \\
& -\gamma_{M}[f(x, p)-f(x,-p)] .
\end{aligned}
$$

This equation is solved numerically with the boundary condition which assumes that the states of conduction electrons inflowing to the nanosystem depend on the states of the charge reservoirs as follows (Frensley, 1990):

$$
\begin{gathered}
\left.f(0, p)\right|_{p>0}=f^{L}(E(p)), \\
\left.f(L, p)\right|_{p<0}=f^{R}(E(p)),
\end{gathered}
$$

where $f^{L(R)}(E(k))$ are the supply functions for the left $(L)$ and right $(R)$ contact in the form

$$
\begin{aligned}
& f^{L(R)}(E(k)) \\
& \quad=\frac{m}{\pi \hbar^{2} \beta} \ln \left\{\exp \left[-\left(\frac{\hbar^{2} k^{2}}{2 m}-\mu_{F}^{L(R)}\right) \beta\right]+1\right\},
\end{aligned}
$$

$p=\hbar k, \beta=1 / k_{B} T, k_{B}$ being the Boltzmann constant and $T$ the temperature, while $\mu_{F}^{L(R)}$ is the Fermi level in the left (right) contact and $\mu_{F}^{L}=\mu_{F}^{R}+e V_{B}$, where $V_{B}$ is the applied bias voltage.

Solving the kinetic equation in the form given by Eqn. 31 with the boundary condition (32) allows determining the distribution function for a given value of the employed bias voltage $V_{B}$. Then the electronic current, $I$, as a function of the bias voltage, is calculated in accordance with the formula

$$
I\left(V_{B}\right)=\frac{e}{2 \pi \hbar L} \int \mathrm{d} x \int \mathrm{d} p \frac{p}{m} f\left(x, p ; V_{B}\right) .
$$




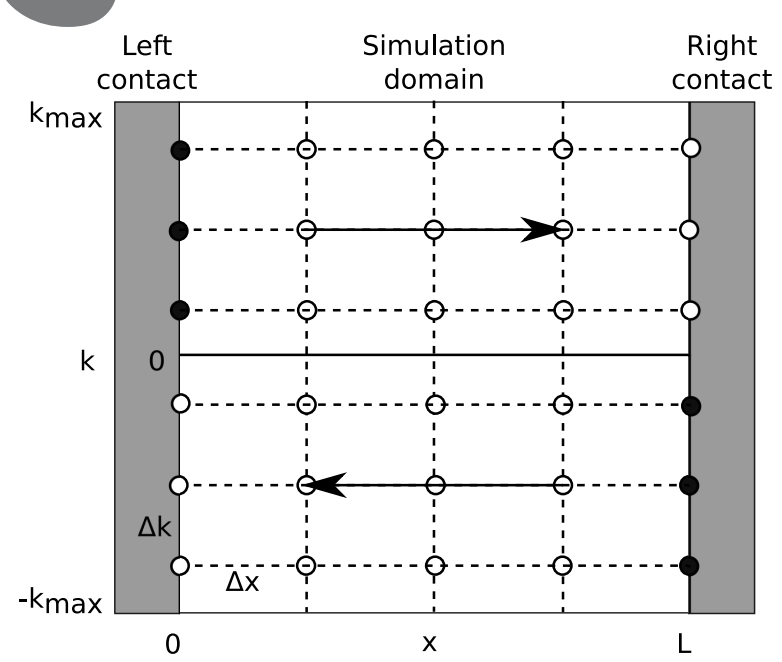

Fig. 2. Discretization scheme for the kinetic equation. Closed circles correspond to the sites at which the fixed boundary condition based on the supply function is applied.
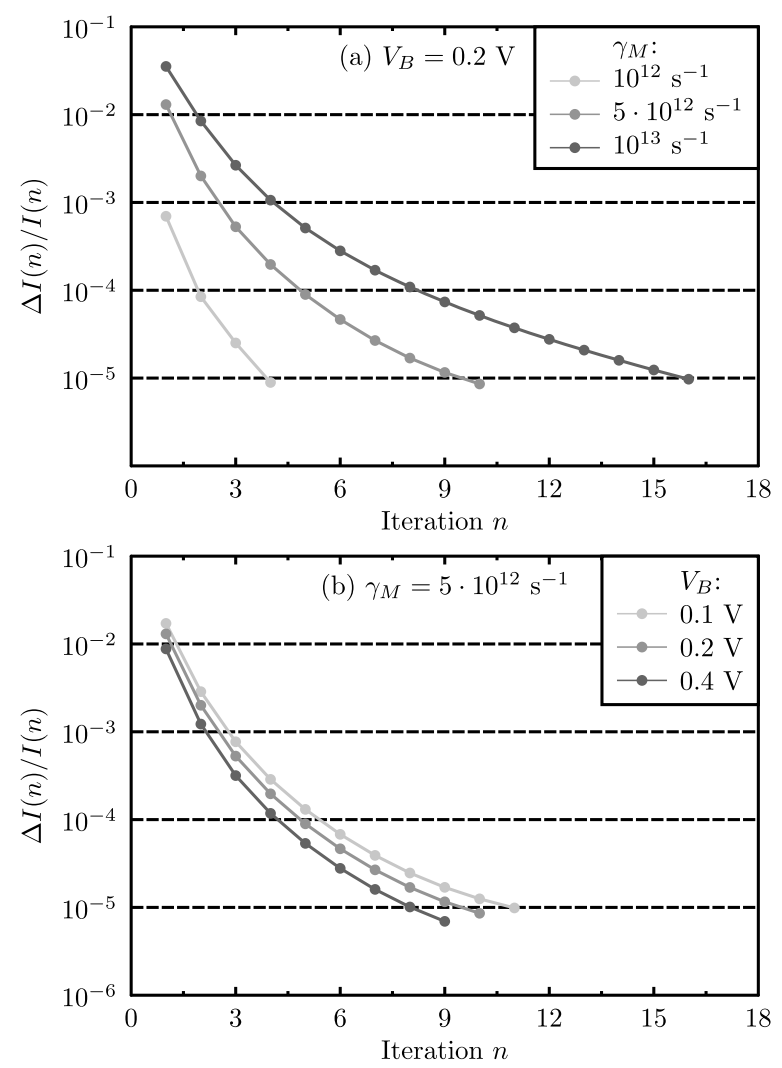

Fig. 3. Illustration of the convergence of the self-consistent procedure used for solving the kinetic equation, in terms of the relative change of the current value between the consecutive iterations, calculated for $V_{B}=0.2 \mathrm{~V}$ and $\gamma_{M}=10^{12}, 5 \cdot 10^{12}, 10^{13} \mathrm{~s}^{-1}(\mathrm{a}), \gamma_{M}=5 \cdot 10^{12} \mathrm{~s}^{-1}$ and $V_{B}=0.1,0.2,0.4 \mathrm{~V}$ (b). In both cases, $\gamma_{R}=0$.

The kinetic equation 31) is solved numerically using the implicit Euler method, with all the following equations written in the atomic units (a.u.) for which it is assumed that

$$
\hbar=e=m_{e}=1,
$$

and therefore $p=k$. The phase space is discretized into $N_{x} \times N_{k}$ cells, each of them having volume $\Delta_{x} \Delta_{k}$, where

$$
\Delta_{x}=\frac{L}{N_{x}}, \quad \Delta_{k}=\frac{2 k_{\max }}{N_{k}} .
$$

In the first step, this procedure requires substituting the derivatives of the distribution function with their three-point discrete approximations resulting from the finite difference method,

$$
\begin{array}{r}
\frac{\mathrm{d}}{\mathrm{d} x} f(x, k) \approx \frac{1}{2 \Delta_{x}}\left(-3 f_{i, j}+4 f_{i+1, j}-f_{i+2, j}\right) \\
\text { for } k_{j}>0
\end{array}
$$

$$
\begin{array}{r}
\frac{\mathrm{d}}{\mathrm{d} x} f(x, k) \approx \frac{1}{2 \Delta_{x}}\left(3 f_{i, j}-4 f_{i-1, j}+f_{i-2, j}\right) \\
\text { for } k_{j}<0
\end{array}
$$

where $f_{i, j}=f\left(x_{i}, k_{j}\right)$ with

$$
\begin{cases}x_{i}=i \Delta_{x}, & i=0, \ldots, N_{x}-1, \\ k_{j}=\left(j-\frac{N_{k}-1}{2}\right) \Delta_{k}, & j=0, \ldots, N_{k}-1 .\end{cases}
$$

It should be noted that the above equations take the asymmetric form, which is a result of the assumed boundary condition, i.e., the right-hand or the left-hand form is chosen depending on the sign of $k$ since the boundary condition is defined at $x=0$ for $k>0$ and at $x=L$ for $k<0$. Additionally, $N_{k}$ must be even, so that the mesh points are defined only for positive or negative values of $k$, and $k=0$ is omitted as shown in Fig. 2. This also means that, if $f_{i, j}$ corresponds to a certain $f(x, k)$, then $f_{i, N_{k}-j-1}$ corresponds to $f(x,-k)$. As a result, we obtain the following equations,

$$
\begin{array}{r}
f_{i, j} \\
=\frac{4 f_{i-1, j}-f_{i-2, j}+2 \frac{m \Delta_{x}}{\hbar k_{j}}\left(\gamma_{R} f_{j}^{e q}+\gamma_{M} f_{i, N_{k}-j-1}\right)}{3+2 \frac{m \Delta_{x}}{\hbar k_{j}}\left(\gamma_{R}+\gamma_{M}\right)} \\
\text { for } k_{j}>0, \quad(40)
\end{array}
$$

$$
\begin{gathered}
f_{i, j} \\
=\frac{4 f_{i+1, j}-f_{i+2, j}-2 \frac{m \Delta_{x}}{\hbar k_{j}}\left(\gamma_{R} f_{j}^{e q}+\gamma_{M} f_{i, N_{k}-j-1}\right)}{3-2 \frac{m \Delta_{x}}{\hbar k_{j}}\left(\gamma_{R}+\gamma_{M}\right)}, \\
\text { for } k_{j}<0 . \quad \text { (41) }
\end{gathered}
$$

They are solved recursively, assuming that $f_{i, N_{k}-j-1}$ is initially equal to the value of the equilibrium distribution 
function, $f_{N_{k}-j-1}^{e q}$. After finding the values of the distribution function at all sites, the whole procedure is repeated in the self-consistent manner until the solution converges, which is verified in terms of the relative change of the current value calculated at the consecutive steps $n-1$ and $n$,

$$
\Delta I(n)=|I(n)-I(n-1)|,
$$

with $\Delta I(n) / I(n)$ required to be less than $10^{-5}$. Typically no more than 20 steps are needed for the solution to converge, as illustrated in Fig. 3 .

\section{Results and a discussion}

The numerical calculations were performed for a system of length $L=60 \mathrm{~nm}$ and at temperature $T=10 \mathrm{~K}$. The Fermi level at the right contact was assumed to be equal to $\mu_{F}^{R}=0.08 \mathrm{eV}$. All calculations were conducted on a computational grid of size $N_{x} \times N_{k}$ with $N_{x}=60$ and $N_{k}=60$, while $\Delta_{x}=1 \mathrm{~nm}$ and $\Delta_{k}=0.04 \mathrm{~nm}^{-1}$.

The scattering rate provides us with information how frequently electrons participate in the elastic or inelastic scattering processes. The higher scattering rate results therefore in higher resistance of the system, which reduces the measured electronic current. This dependence, calculated for the system considered, is presented in Fig. 4, which shows how the current-voltage characteristics of the simulated structure react to the change of the scattering rates.

These results show that both types of scattering have noticeable impact on the current, while the influence of elastic scattering is clearly stronger. This kind of transport characteristics can be observed in defected semiconductor thin films at higher temperatures. One of the examples of such materials is titanium dioxide, which belongs to a class of wide-band gap insulators. The progressive transition from an insulator to semiconductor can be accomplished by introduction of point defects which are responsible for semiconducting properties of $\mathrm{TiO}_{2-x}$. In an atmosphere of low oxygen activity, titanium dioxide becomes an oxygen-deficient semiconductor due to the electronic disorder related to nonstoichiometry $x: \mathrm{TiO}_{2}$ $\leftrightarrow x / 2 \mathrm{O}_{2}+\mathrm{TiO}_{2-x}$. The point defects proposed in the literature are oxygen vacancies, titanium interstitials or a combinations of these. All of them form shallow donor levels (Stashans et al., 1996; Nowotny et al., 1997; Bak et al., 2006). Their ionization provides electrons to conduction band necessary for electrical conduction, which can be observed even at room temperature for nonstoichiometric $\mathrm{TiO}_{2-x}$. Figure 5 displays results of current-voltage characteristics measurements for $\mathrm{TiO}_{2}$ at $T=300 \mathrm{~K}$ and different intensity ratios $I / I_{0}$.

The nonstoichiometric $\mathrm{TiO}_{2-x}$ thin films were deposited by dc-pulsed magnetron sputtering from a metallic Ti target in the reactive $\mathrm{Ar}+\mathrm{O}_{2}$ atmosphere with
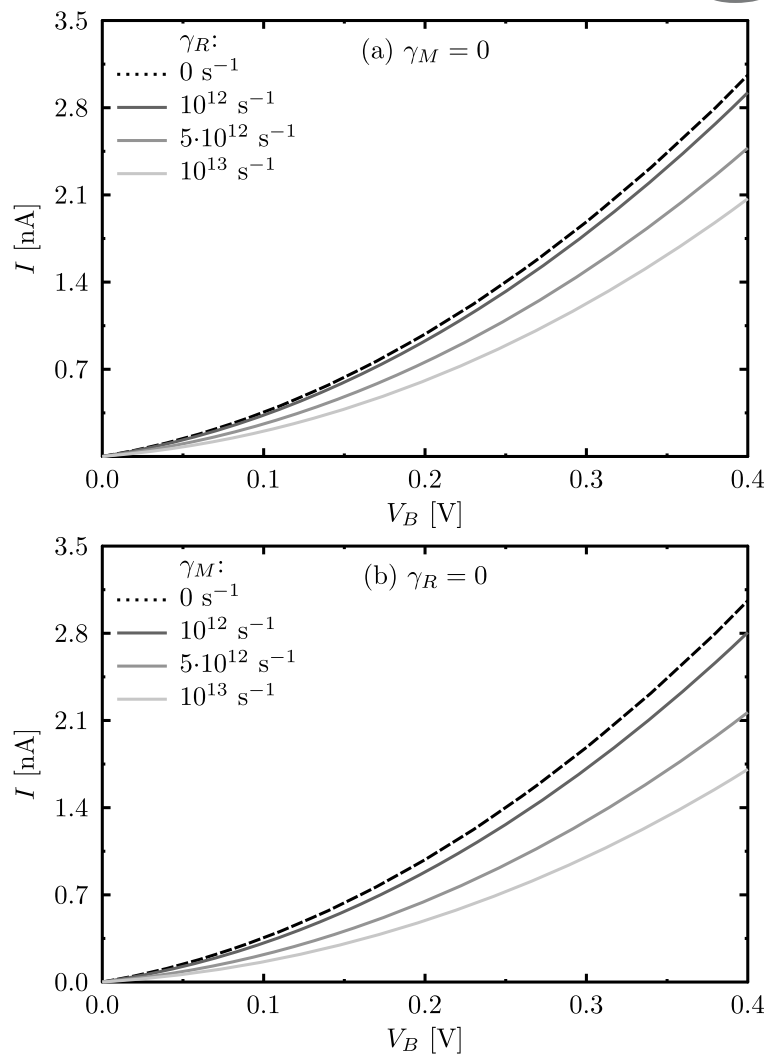

Fig. 4. Current voltage characteristics for different inelastic scattering rates dissipating energy (a) and various elastic scattering rates responsible for momentum randomization (b).

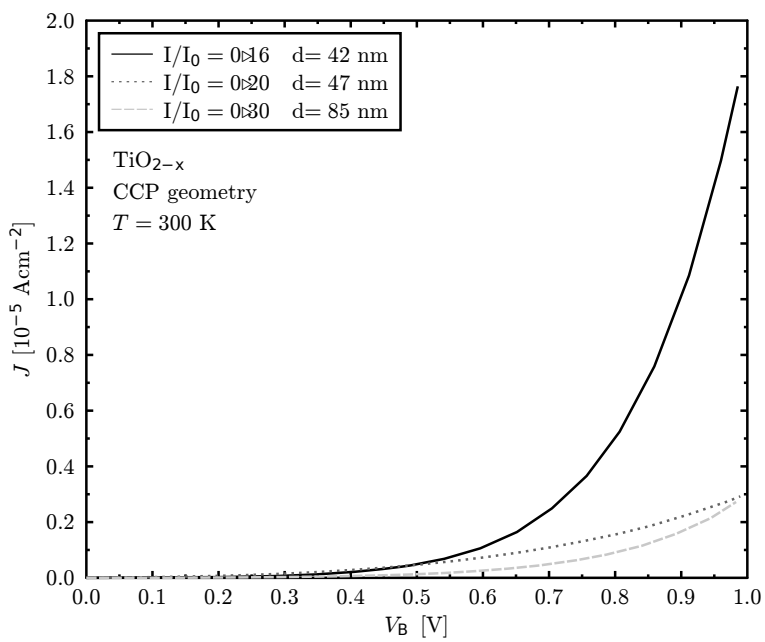

Fig. 5. Current-voltage characteristics measured for $\mathrm{TiO}_{2}$ at different intensity ratios $I / I_{0}$ and thin film thicknesses $d$.

optical control. The optical control system is based on plasma emission and uses the relative ratio of the intensity $I / I_{0}$ of the Ti emission line as the parameter that controls the departure from stoichiometry $x$ in $\mathrm{TiO}_{2-x}$. The line 

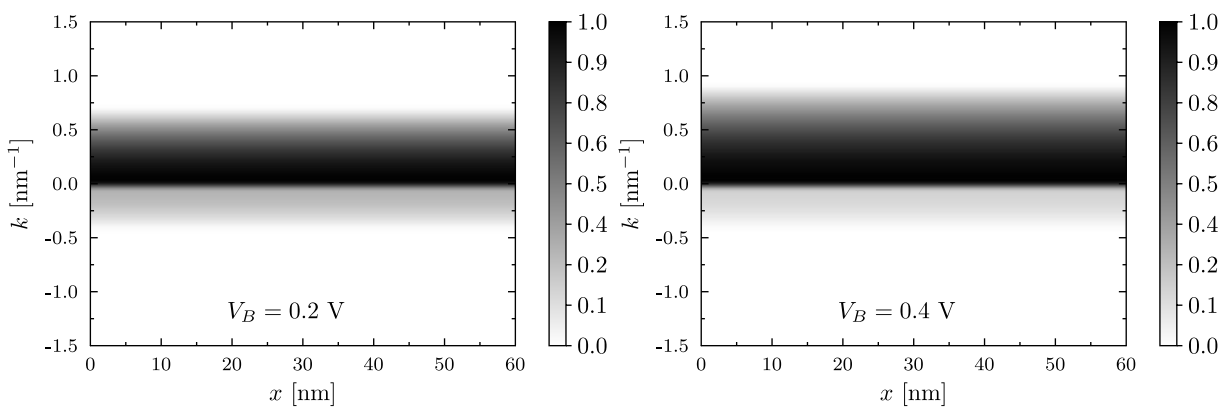

(a)
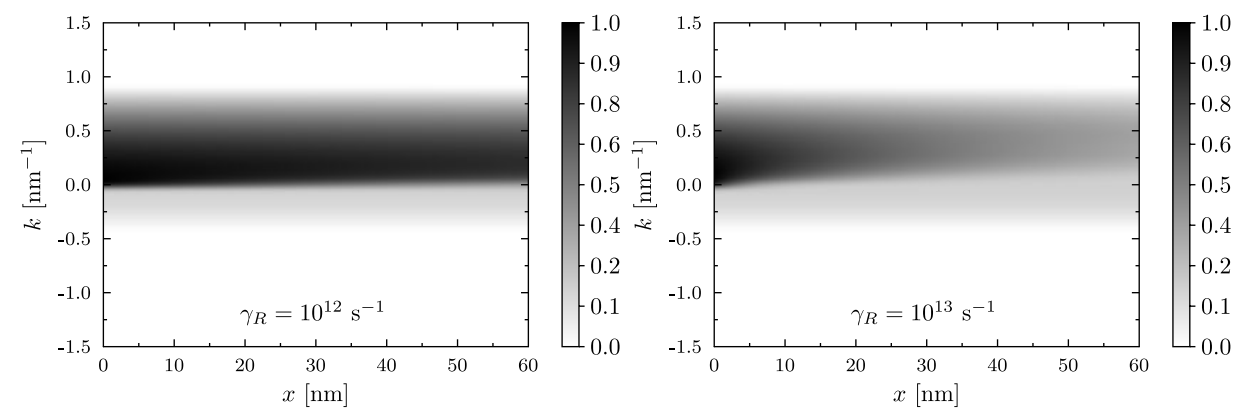

(b)
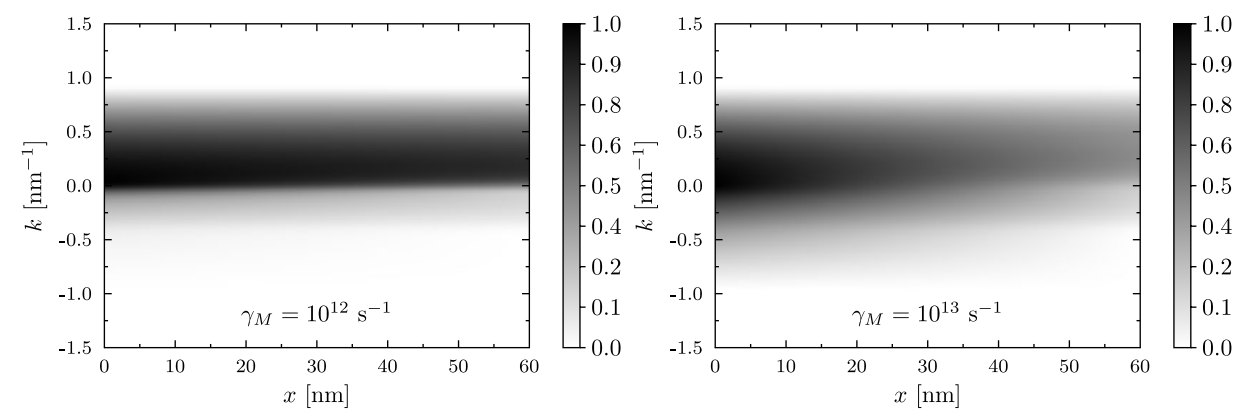

(c)

Fig. 6. Normalized distribution function $f(x, k)$ for $\gamma_{R}=\gamma_{M}=0$ at the bias voltage $V_{B}=0.2 \mathrm{~V}$ and $V_{B}=0.4 \mathrm{~V}$ (a), $\gamma_{M}=0 \mathrm{~s}^{-1}$, $\gamma_{R}=10^{12} \mathrm{~s}^{-1}$ and $\gamma_{R}=10^{13} \mathrm{~s}^{-1}$ at the bias voltage $V_{B}=0.4 \mathrm{~V}(\mathrm{~b})$, and $\gamma_{R}=0 \mathrm{~s}^{-1}, \gamma_{M}=10^{12} \mathrm{~s}^{-1}$ and $\gamma_{M}=10^{13} \mathrm{~s}^{-1}$ at the bias voltage $V_{B}=0.4 \mathrm{~V}$ (c).

intensity $I_{0}$ corresponds to pure metallic deposition mode at $100 \%$ Ar. The intensity $I$ of the Ti emission line at $\lambda=500 \mathrm{~nm}$ at a given $\mathrm{Ar}+\mathrm{O}_{2}$ reflects the oxidation state of the titanium target. The increase in $I / I_{O}$ is followed by an increasing departure from stoichiometry. Thin films with a thickness of 40-80 nm were deposited onto ITO glass at the substrate temperature $523 \mathrm{~K}$. These results are consistent with the present calculations. Defects in the systems considered can be treated as the scattering centers for conduction electrons. Because the number of defects increases with the ratio of the intensity $I / I_{0}$, the scattering processes are more efficient and lead to the decrease of the relaxation time, thereby the resistance of the thin layer increases.

The source of this behavior is a big change in the distribution function due to the dissipation processes. Examples of the calculated distribution functions are presented in Fig. 6, which shows the functions $f(x, k)$ that are solutions of the kinetic equation (31). Analysis of those distribution functions clearly shows that larger values of the current are related to bigger carrier density in the upper half of the phase space, i.e., for $k>0$. When the non-zero scattering rates are introduced, the distribution function for both types of scattering is mostly affected for carriers with a positive momentum. For inelastic scattering, the distribution function changes only for $k>0$, and its values decrease until reach the same values as for $k<0$, which are equal to the distribution function in an equilibrium. For elastic scattering, values of distribution function decrease for $k>0$, and increase 


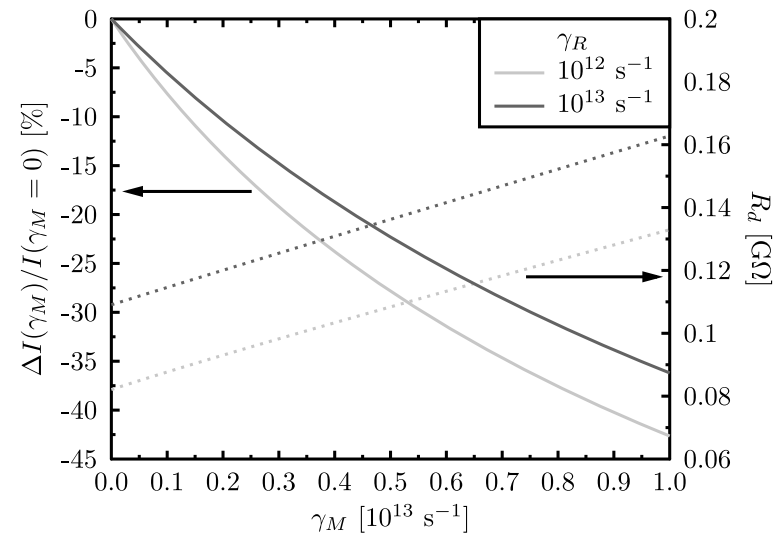

Fig. 7. Relative change of the current (solid lines) and differential resistance (dashed lines) due to the influence of the dissipation processes calculated for different scattering rates and the bias voltage $V_{B}=0.4 \mathrm{~V}$.

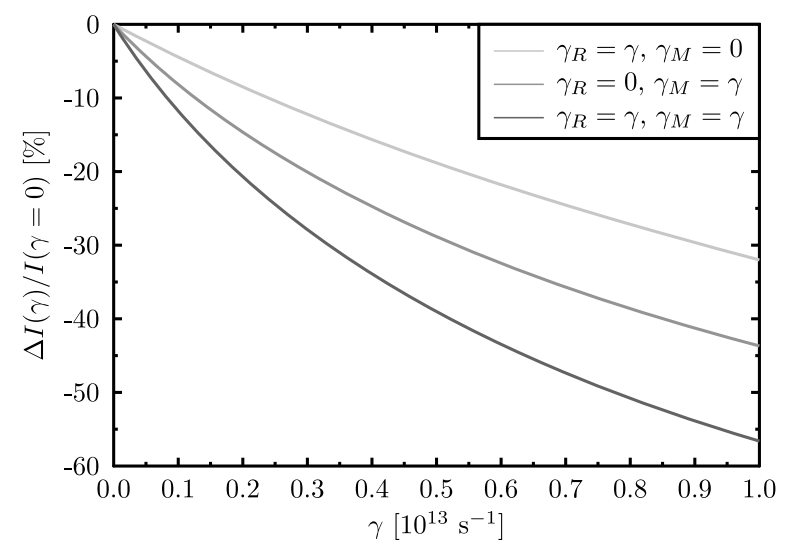

Fig. 8. Relative change of the current caused by different dissipation processes calculated for different scattering rates and the bias voltage $V_{B}=0.4 \mathrm{~V}$.

for $k<0$, which causes a drop in the current bigger than for inelastic scattering. As a result, the introduction of scattering changes the value of the current, and modifies the shape of the distribution function which changes in a much smoother way around $k=0$.

The influence of scattering processes on the behaviour of the analyzed system can be also illustrated by the relation between the scattering rate and the relative difference between the current flowing in absence of any dissipation processes and the current calculated in presence of elastic or inelastic scattering. Figures 7 and 8 confirm that the relative change in the current due to the dissipation processes depends on the type of scattering which is taken into account. The relative change in the current resulting from elastic scattering, presented in Fig. 7 was calculated as $\Delta I\left(\gamma_{M}\right) / I\left(\gamma_{M}=0\right)$, where $\Delta I\left(\gamma_{M}\right)=I\left(\gamma_{M}\right)-I\left(\gamma_{M}=0\right)$, with $\gamma_{R}$ treated as a constant and non-zero parameter different for each line in
Fig. 7. It shows that the increase in $\gamma_{M}$ reduces the current flowing through the system, but this change is noticeably smaller if a larger value of $\gamma_{R}$ is assumed. This impact of $\gamma_{R}$ on the change in the current indicates that it suppresses the impact of $\gamma_{M}$ on transport characteristics. Also the differential resistance, $R_{d}=\mathrm{d} V_{B} / \mathrm{d} I$, is sensitive to the scattering, as shown for $V_{B}=0.4 \mathrm{~V}$ in Fig. 7. Its value increases linearly with increasing $\gamma_{M}$, and at all points is slightly larger for greater $\gamma_{R}$. In Fig. 8 it is shown that the relative current change due to inelastic and elastic scattering is a nonlinear, increasing function of the scattering rate equal up to approximately $30 \%$ for $\gamma_{R}=10^{13} \mathrm{~s}^{-1}$, over $45 \%$ for $\gamma_{M}=10^{13} \mathrm{~s}^{-1}$ and over $55 \%$ for $\gamma_{R}=\gamma_{M}=10^{13} \mathrm{~s}^{-1}$.

\section{Conclusions}

We solved numerically the kinetic equation with the dissipative term which is modeled by the relaxation time approximation, with separate terms describing the relaxation of the momentum and the relaxation of the energy. The numerical solution of the kinetic equation allowed us to find the distribution function. We investigated the modification of the shape of this function due to those two types of scattering. Owing to the knowledge of the distribution functions, also the electronic current was calculated as a function of the bias voltage for different values of scattering rates corresponding to the term which dissipates energy and to the momentum-relaxation term. Our calculations show that the current-voltage characteristics are considerably modified by elastic and inelastic scattering, and the results of the experiments which we performed for defected semiconductor thin films exhibit the same shape of the current-voltage characteristics as the numerical results. Furthermore, the comparison of the experimental data and the results of the calculations proves that the changes in the intensity ratio and the film thickness have similar impact on the current-voltage characteristics as the elastic/inelastic scattering rates used in our theoretical model.

\section{Acknowledgment}

The authors are grateful to A. Brudnik from the Department of Electronics, Faculty of Electrical Engineering, Automatics, Computer Science and Electronics, AGH-UST, for preparation of dc magnetron sputtered samples.

This work was partially supported under the Faculty of Physics and Applied Computer Science AGH UST statutory tasks, within the subsidy of Ministry of Science, and Higher Education. Karol Kulinowski was partly supported by the EU project POWR.03.02.00-00-I004/16.

The primary version of this paper was presented at 
the 3rd Conference on Information Technology, Systems Research and Computational Physics, Cracow, Poland, 2018.

\section{References}

Bak, T., Nowotny, J. and Nowotny, M.K. (2006). Defect disorder of titanium dioxide, Journal of Physical Chemistry B 110(43): 21560-21567.

Ben Abdallah, N., Degond, P. and Genieys, S. (1996). An energy-transport model for semiconductors derived from the Boltzmann equation, Journal of Statistical Physics 84(1): 205-231.

Cabrera, R., Bondar, D.I., Jacobs, K. and Rabitz, H.A. (2015). Efficient method to generate time evolution of the Wigner function for open quantum systems, Physical Review A 92(4): 042122.

Caldeira, A.O. and Leggett, A.J. (1981). Influence of dissipation on quantum tunneling in macroscopic systems, Physical Review Letters 46(4): 211-214.

Chatterjee, K., Roadcap, J.R. and Singh, S. (2014). A new Green's function Monte Carlo algorithm for the solution of the two-dimensional nonlinear Poisson-Boltzmann equation: Application to the modeling of the communication breakdown problem in space vehicles during re-entry, Journal of Computational Physics 276: 479-485.

Chruściński, D. and Pascazio, S. (2017). A brief history of the GKLS equation, Open Systems and Information Dynamics 24(3): 1740001.

Costolanski, A.S. and Kelley, C.T. (2010). Efficient solution of the Wigner-Poisson equations for modeling resonant tunneling diodes, IEEE Transactions on Nanotechnology 9(6): 708-715.

Danielewicz, P. (1984). Quantum theory of nonequilibrium processes I, Annals of Physics 152(2): 239-304.

Di Ventra, M. (2008). Electrical Transport in Nanoscale Systems, Cambridge University Press, Cambridge.

Ferry, D.K., Goodnick, S.M. and Bird, J. (2009). Transport in Nanostructures, Cambridge University Press, Cambridge.

Frensley, W.R. (1990). Boundary conditions for open quantum systems driven far from equilibrium, Reviews of Modern Physics 62(3): 745-791.

Fujita, S. (1966). Introduction to Non-Equilibrium Quantum Statistical Mechanics, W.B. Saunders Company, Philadelphia, PA.

Gómez, E.A., Thirumuruganandham, S.P. and Santana, A. (2014). Split-operator technique for propagating phase space functions: Exploring chaotic, dissipative and relativistic dynamics, Computer Physics Communications 185(1): 136-143.

Hong, S. and Jang, J. (2018). Transient simulation of semiconductor devices using a deterministic Boltzmann equation solver, IEEE Journal of the Electron Devices Society 6: 156-163.
Hong, S.-M., Pham, A.-T. and Jungemann, C. (2011). Deterministic Solvers for the Boltzmann Transport Equation, Springer Science \& Business Media, Vienna.

Jacoboni, C., Bertoni, A., Bordone, P. and Brunetti, R. (2001). Wigner-function formulation for quantum transport in semiconductors: Theory and Monte Carlo approach, Mathematics and Computers in Simulation 55(1-3): 67-78.

Jacoboni, C., Poli, P. and Rota, L. (1988). A new Monte Carlo technique for the solution of the Boltzmann transport equation, Solid-State Electronics 31(3): 523-526.

Jonasson, O. and Knezevic, I. (2015). Dissipative transport in superlattices within the Wigner function formalism, Journal of Computational Electronics 14(4): 879-887.

Kim, K.-Y. (2007). A discrete formulation of the Wigner transport equation, Journal of Applied Physics 102(11): 113705.

Kim, K.-Y. and Kim, S. (2015). Effect of uncertainty principle on the Wigner function-based simulation of quantum transport, Solid-State Electronics 111: 22-26.

Kohn, W. and Luttinger, J.M. (1957). Quantum theory of electrical transport phenomena, Physical Review 108(3): 590-611.

Kulczycki, P., Kacprzyk, J., Kóczy, L., Mesiar, R. and Wisniewski, R. (2019). Information Technology, Systems Research, and Computational Physics, Springer, Cham, (in press).

Kulczycki, P., Kowalski, P. and Łukasik, S. (Eds) (2018). Contemporary Computational Science, AGH-UST Press, Cracow, p. 4.

Leaf, B. (1968). Weyl transformation and the classical limit of quantum mechanics, Journal of Mathematical Physics 9(1): 65-72.

Lee, H.-W. (1995). Theory and application of the quantum phase-space distribution functions, Physics Reports 259(3): 147-211.

Lifshits, I.M., Gredeskul, S.A. and Pastur, L.A. (1987). Introduction to the Theory of Disordered Systems, John Wiley and Sons, Inc., New York, NY.

Luttinger, J.M. and Kohn, W. (1958). Quantum theory of electrical transport phenomena II, Physical Review 109(6): 1892-1909.

Mahan, G.D. (2000). Many Particle Physics, Kluwer Academic Plenum Publishers, New York, NY.

Muscato, O. and Wagner, W. (2016). A class of stochastic algorithms for the Wigner equation, SIAM Journal on Scientific Computing 38(3): A1483-A1507.

Nedjalkov, M., Kosina, H., Selberherr, S., Ringhofer, C. and Ferry, D.K. (2004). Unified particle approach to Wigner-Boltzmann transport in small semiconductor devices, Physical Review B 70(11): 115319.

Nedjalkov, M., Selberherr, S., Ferry, D., Vasileska, D., Dollfus, P., Querlioz, D., Dimov, I. and Schwaha, P. (2013). Physical scales in the Wigner-Boltzmann equation, Annals of Physics 328: 220-237. 
Nedjalkov, M. and Vitanov, P. (1989). Iteration approach for solving the Boltzmann equation with the Monte Carlo method, Solid-State Electronics 32(10): 893-896.

Nowotny, J., Radecka, M. and Rekas, M. (1997). Semiconducting properties of undoped $\mathrm{TiO}_{2}$, Journal of Physics and Chemistry of Solids 58(6): 927-937.

Querlioz, D. and Dollfus, P. (2010). The Wigner Monte-Carlo Method for Nanoelectronic Devices: A Particle Description of Quantum Transport and Decoherence, ISTE Ltd. and John Wiley and Sons, Inc., New York, NY.

Rammer, J. (2007). Quantum Field Theory of Non-Equilibrium States, Cambridge University Press, Cambridge.

Schieve, W.C. and Horwitz, L.P. (2009). Quantum Statistical Mechanics, Cambridge University Press, Cambridge.

Schleich, W.P. (2001). Quantum Optics in Phase Space, John Wiley and Sons, Inc., New York, NY.

Schulz, D. and Mahmood, A. (2016). Approximation of a phase space operator for the numerical solution of the Wigner equation, IEEE Journal of Quantum Electronics 52(2): $1-9$.

Sellier, J., Amoroso, S., Nedjalkov, M., Selberherr, S., Asenov, A. and Dimov, I. (2014). Electron dynamics in nanoscale transistors by means of Wigner and Boltzmann approaches, Physica A: Statistical Mechanics and Its Applications 398: 194-198.

Sellier, J. and Dimov, I. (2014). The Wigner-Boltzmann Monte Carlo method applied to electron transport in the presence of a single dopant, Computer Physics Communications 185(10): 2427-2435.

Spisak, B.J., Wołoszyn, M. and Szydłowski, D. (2015). Dynamical localisation of conduction electrons in one-dimensional disordered systems, Journal of Computational Electronics 14(4): 916-921.

Stashans, A., Lunell, S. and Grimes, R.W. (1996). Theoretical study of perfect and defective $\mathrm{TiO}_{2}$ crystals, Journal of Physics and Chemistry of Solids 57(9): 1293-1301.

Tatarski1̌, V.I. (1983). The Wigner representation of quantum mechanics, Soviet Physics Uspekhi 26(4): 311-327.

Ter Haar, D. (1961). Theory and applications of the density matrix, Reports on Progress in Physics 24(1): 304-362.

Thomann, A. and Borz, A. (2017). Stability and accuracy of a pseudospectral scheme for the Wigner function equation, Numerical Methods for Partial Differential Equations 33(1): 62-87.
Wigner, E. (1932). On the quantum correction for thermodynamic equilibrium, Physical Review 40(5): 749-759.

Zurek, W.H. (2003). Decoherence, einselection, and the quantum origins of the classical, Reviews of Modern Physics 75(3): 715-775.

Karol Kulinowski received his BSc degree in technical physics from the AGH University of Science and Technology, Cracow, Poland, in 2016, and his MSc degree in nanotechnology from the the Wrocław University of Science and Technology, Wrocław, Poland, in 2017. Currently he is a PhD student at the AGH-UST. His main research interests are in simulation of electron transport properties in semiconductors using the Wigner function formalism and numerical calculations.

Maciej Wołoszyn received his $\mathrm{PhD}$ degree in physics from the $\mathrm{AGH}$ University of Science and Technology, Cracow, Poland, in 2006. Currently he is an assistant professor there. His main research interests are in simulation of electron transport properties in semiconductors using the Wigner function formalism and numerical calculations.

Marta Radecka received her $\mathrm{PhD}$ and $\mathrm{DSc}$ degrees in chemistry from the AGH University of Science and Technology, Cracow, Poland, in 1993 and 2004, respectively. She is currently a full professor of chemistry there. Her research interests include the development of photoelectrochemical cells, semiconducting gas sensors and experimental studies of the electrical and structural properties of ceramic oxides and thin films, as well as electron transport mechanisms in semiconductors. Her research works attempt to understand the correlation between the defect structure of crystals and physico-chemical properties of nonstoichiometric compounds.

Bartłomiej J. Spisak received his PhD and DSc degrees in theoretical condensed matter physics from the AGH University of Science and Technology, Cracow, Poland, in 2003 and 2014, respectively. He is currently an assistant professor there. His main research interests concern the theoretical description of transport properties of condensed matter systems, the quasi-distribution function formalism in the phase space and random matrix theory.
Received: 15 October 2018

Revised: 1 February 2019

Accepted: 18 March 2019 